\title{
Introduction to the Minitrack on Digital Innovation, Transformation, and Entrepreneurship
}

\author{
Nicholas Berente \\ University of Notre Dame \\ nberente@,nd.edu
}

\author{
Kalle Lyytinen \\ Case Western Reserve \\ University \\ kalle@,case.edu
}

\author{
Youngjin Yoo \\ Case Western Reserve \\ University \\ youngjin.yoo@case.edu
}

In the pervasive digital epoch, organizations must continually innovate with digital technologies in order to succeed. This innovation involves generation of new products and services using digital assets (digital innovation), fundamental changes to organizing (digital transformation), and fostering of new ventures through digital innovations (digital entrepreneurship). New products, services, operational models, business models, industrial arrangements, work organization, etc., have all been dramatically influenced by digital technologies that are involved in their development, and the digital technologies that are embedded in the innovations themselves.

This minitrack offers a venue for research that focuses on digital technologies and associated forms of organizational innovation, broadly conceived. This encompasses research into the antecedents, processes, infrastructures, and outcomes associated with the generation and appropriation of novel product and service innovations enabled by digital technologies. Such innovations enable new forms of organizing, including new business models and work arrangements of all sorts. Existing organizations are undergoing digital transformations as they try out new organizational structures and processes enabled by digitalization. New ventures, either within those organizations or as startups, manifest in varying forms of digital entrepreneurship. Due to its scope the minitrack aligns with the AIS Special Interest Group on Digital Innovation, Transformation, and Entrepreneurship (SIG-DITE).

Next we briefly describe these three broad themes of the minitrack.

\section{Digital Innovation}

Digital technologies form essential components of many of contemporary novel product and service offerings. Organizations need to digitally-enable their products, platformize their services, and find alternative ways to plug into existing and emerging digital ecosystems. Their products and services need to continually leverage wave upon wave of nextgeneration digital technologies (e.g., robots, 3D printing, sensor networks, blockchain, quantum computing, etc., etc.) and engage with existing install base of digital technology such as mobile, social, big data, cloud computing, virtual/augmented reality, Internet of Things.

Further, these digital technologies, tools, platforms, and associated infrastructures that support underlying innovative processes including software development, product design \& engineering, R\&D, and science are becoming ubiquitous. Organizational practices surrounding these processes may be different from organizational practices of the past because they involve extensive virtual collaboration, geographic distribution, iteration, simulation, and related novel generative activities. They often follow radically different temporal pattern - often expressed as an "agile" method. The tools and infrastructures upon which these practices rest currently shape those practices across manifold industrial contexts from financial services, healthcare, manufacturing to big pharma.

Another key area is how established organizations can not only start new digital innovations, but scale them into robust businesses is another emerging topic. Challenges around who should be involved in the creation and scaling of these new digital business lines are key strategic challenges of many established organizations who started the digital innovations early.

\section{Digital Transformation}

As organizations increasingly leverage such digital technologies, they must transition to new ways of thinking about their business and value creation and capture. Digital technologies can have a tremendous impact on organizational structure and strategy. This 
is reflected in recent growing streams of research on digital architectures, modularity, platforms, and system architectures. Widespread digital capabilities and associated practices enable and undergird a variety of novel business models. Topics such as digital disruption of traditional industries, digital transformation of incumbent organizations, and digital convergence of technologies and ecosystems are all at the forefront of innovation around business models. Furthermore such changes in organizations also changes industry architecture and broader institutional and regulatory regimes.

Advancements in digital technologies have rendered possible new ways of doing things within and among organizations. These unprecedented ways of doing things adopt often the form of process innovations that have the potential to transform organizational tasks and associated organizing.

\section{Digital Entrepreneurship}

Digital innovation opens up continual opportunities for entrepreneurial activity. New digital ventures as well as intrapreneurial activities in established organizations are both concerned with designing new innovative businesses that leverage digital technologies. Topics in digital entrepreneurship include new venture formation involving the deployment of digital technologies and digital business models, agile forms of launching new business processes, and uses of digital tools and platforms that promote customer intimacy, design thinking, and lean startup principles. Also financing these entrepreneurial activities leveraging digital technology takes a new dimension in digital entrepreneurship and include new forms of corporate venture, crowd funding, access to new forms of venture capital, private equity, related angel investing, and the relationship of these approaches to incubators, accelerators, and other arrangements that seek to launch and support digital ventures. New forms of collaboration between established firms and digital ventures through these third-party mediators and how they are integrated back into the established firms to fuel internal digital innovations and transformations also demand more careful examinations.

\section{Conclusion}

The minitrack strongly emphasizes the ways in which particular digital technologies contribute to organizational innovation in manifold ways. Papers in the minitrack are expected to heed to the pivotal elements and features of the digital technologies that shape or impact innovation processes and outcomes. 